\title{
Alineación entre Sistemas de Información, Tecnologías de la de Información y el Negocio: un Estudio Empírico en la Región de Biobío, Chile
}

\author{
Sergio A. Araya-Guzmán(1), Guido Hernández-Uribe ${ }^{(1)}$ y Patricio E. Ramírez-Correa(2)* \\ (1) Universidad del Bío-Bío, Facultad de Ciencias Empresariales, Avenida Collao 1202, Concepción, Chile. \\ (e-mail: saraya@ubiobio.cl; guido.hernandez@hotmail.cl) \\ (2) Escuela de Ingeniería, Universidad Católica del Norte, Larrondo 1281, Coquimbo, Chile. \\ (e-mail: patricio.ramirez@ucn.cl).
}

${ }^{*}$ Autor a quien debe ser dirigida la correspondencia

Recibido Oct. 12, 2018; Aceptado Dic. 18, 2018; Versión final Mar. 16, 2019, Publicado Oct. 2019

\begin{abstract}
Resumen
Este trabajo estudia el efecto de las capacidades asociadas con Sistemas y Tecnologías de Información (SI/TI) en la alineación de SI/TI con la estrategia de negocio. Para estos efectos, se aplicó una encuesta a responsables de $\mathrm{SI} / \mathrm{TI}$ y a directivos que tuvieran alguna influencia en las decisiones relacionadas con los $\mathrm{SI} / \mathrm{TI}$, pertenecientes a empresas de la Región del Biobío (Chile). Las variables se determinaron utilizando escalas de medida de estudios previos. El análisis de los datos se realizó por medio de modelo de ecuaciones estructurales basado en mínimos cuadrados parciales (PLS, Partial Least Squares). Los resultados indican que la alineación se explica en un 52\% por las capacidades asociadas a SI/TI. Se concluye que, si bien el desarrollo e implementación de sistemas y tecnologías de información requiere el apoyo de la alta dirección, es fundamental la participación de los directivos SI/TI para el logro de la alineación.
\end{abstract}

\section{Alignment between Information Systems, Information Technology and Business: An Empirical Study in the Biobío Region (Chile)}

\begin{abstract}
This paper studies the effect of the capacities associated with Information Systems and Technologies (IS/IT) in the alignment of IS/IT with the business strategy. For these purposes, a survey was applied to managers of IS/IT and managers who that have some influence in the decisions related to the IS/IT, belonging to companies of the Biobío Region (Chile). The variables were determined by using measurement scales from previous studies. The analysis of the data was carried out by means of structural equations model based on PLS (Partial Least Squares). The results indicate that the alignment is explained in $52 \%$ by the capacities associated with IS/IT. It is concluded that, although the development and implementation of information systems and technologies requires the support of top management, the participation of the IS/IT managers for the achievement of the alignment is fundamental.
\end{abstract}

Keywords: business-technology alignment; information technology; Partial Least Squares 


\section{INTRODUCCIÓN}

Los Sistemas de Información (SI) son considerados herramientas de potencial estratégico ya que pueden ser de utilidad para el desarrollo organizacional (Valencia, Marulanda y López, 2018), para mejorar y fortalecer la eficiencia y agilidad en las organizaciones (Barney, 1991), aportando valor a estas (Nevo y Wade, 2010), colaborando en mejorar su desempeño (Uwizeyemungu y Raymond, 2012; Gu y Jung, 2013; Liu et al., 2013), y generando beneficios organizacionales (Nevo y Wade, 2010; Drnevich y Croson, 2013; Lin et al., 2014). Desde el punto de vista estratégico, mejorar el desempeño de las organizaciones ha sido de constante interés académico (Porter, 1980; Turban et al., 2015), lo que ha generado el desarrollo de distintos enfoques que abordan su estudio, siendo uno de ellos el análisis de los recursos y capacidades que posee la organización como elementos que permiten establecer una diferencia con la competencia (Barney, 1991), encontrándose entre tales recursos y capacidades aquellos relacionados con los Sistemas y Tecnologías de Información (SI/TI) (Araya et al., 2007a; Nevo y Wade, 2010; Drnevich y Croson, 2013). La obtención de mejores niveles de desempeño y de ventajas competitivas puede estar motivada por la alineación existente entre los SI/TI y la estrategia de negocio de la organización (Kearns y Lederer, 2000; Cragg, King y Hussin, 2002; Tian et al., 2010). Si bien la validez de esta última propuesta se ha analizado en economías desarrolladas, existe carencia de estudios en un contexto de desarrollo tecnológico no avanzado. En este contexto, este estudio tiene por objetivo estudiar el efecto de las capacidades asociadas a los SI/TI sobre la alineación de estos últimos con la estrategia de negocio.

La búsqueda de ventajas competitivas es un propósito primordial para las empresas, y en esta contante exploración, el uso de tecnologías de información surge como una atractiva alternativa. No obstante lo anterior, no pocas empresas fallan en el desarrollo de ventajas competitivas en base a la tecnología de información. En el marco de la teoría de recursos y capacidades, Barney et al. (2001) sostienen que por ser la tecnología de información un recurso de fácil transferencia no genera por si sola rentas superiores para las empresas, sin embargo, la interacción entre esta tecnología y las habilidades de los usuarios pueden ser inimitables y por tanto ser fuente de ventajas competitivas sostenibles. De hecho, el estudio de Bharadwaj (2000) señala que las empresas con una capacidad reconocida de innovación y dirección de tecnología alcanzan mejores resultados de negocio que su competencia. En este mismo sentido, los hallazgos de Ramírez-Correa y Alfaro-Pérez (2011) demuestran que la sola posesión de tecnología de información por la empresa no es garante de mejores resultados financieros. En este contexto, la literatura señala que a través de un proceso continuo de alinear la Tecnología de la Información (TI) y las estrategias de negocios, las empresas pueden desarrollar estas ventajas competitivas y mejorar su desempeño (Kearns y Lederer, 2000; Croteau y Bergeron, 2001; Turban et al., 2015). Reich y Benbasat (1996) definen alineación como el grado en que la misión, objetivos y planes contenidos en la estrategia del negocio son compartidos y respaldados por la estrategia de tecnologías de información. En la misma línea, Chan et al. (1997) y Cragg, King y Hussin (2002) entienden la alineación estratégica de los SI/TI como la relación o ajuste entre la orientación estratégica que tengan los SI y la orientación estratégica de la organización.

Tian et al. (2010) efectúa un estudio sobre la contribución que pueden realizar las capacidades de TI sobre la obtención de ventajas competitivas, considerando entre otras variables el alineamiento de TI con el negocio, estableciendo que este alineamiento presenta un efecto indirecto, marcado por variables como la flexibilidad estratégica de TI (capacidad de la empresa para responder a diversas demandas de TI en entornos competitivos dinámicos) y la asociación negocio-TI (capacidad de la organización para aunar esfuerzos del área o unidad de TI y de otras áreas o unidades de negocio en la implementación de sistemas de información que constituyan un real apoyo al funcionamiento de la empresa). Kearns y Lederer (2000) estudian el efecto de la alineación estratégica en el uso de los recursos de SI para obtener ventajas competitivas y mejorar su desempeño, y consideran dos modelos de alineación estratégica de SI: (i) alineación del plan de SI con el plan de negocio, y (ii) alineación del plan de negocio con el plan de SI. La primera alineación relaciona directamente el plan de SI con la misión, objetivos y estrategias del plan de negocio, es decir, la misión, los objetivos, y la estrategia del negocio son los que guían el diseño del plan de SI y asegura la alineación con la dirección de la empresa. La segunda alineación se logra cuando el plan de negocio referencia directamente al plan de SI y reconoce, considera o referencia aplicaciones y tecnologías de SI específicas.

De acuerdo con Chan y Reich (2007), existen cuatro dimensiones de alineación. La primera es la dimensión estratégica, que se refiere al grado en que la estrategia y los planes de negocios, y la estrategia y los planes de tecnología de información se complementan mutuamente. La segunda es la dimensión social, la cual está asociada al estado en el que se desarrolla el negocio y cómo los ejecutivos de tecnologías de información entienden y están comprometidos con la misión empresarial y con los objetivos y planes de tecnología de información. La tercera es la dimensión estructural, referida al grado de ajuste estructural entre tecnología de información y el negocio, y asociada tanto a la ubicación de los ejecutivos de tecnologías de información en la empresa, como al grado de centralización y al despliegue del personal de tecnologías de información. Y 
finalmente, la dimensión cultural, asociada al importante ajuste cultural entre las empresas y las tecnologías de información, como una precondición para el éxito en planificación de tecnologías de información.

Las consecuencias de la alineación asociadas al desarrollo de ventajas competitivas se pueden detallar, en un sentido descriptivo, a través de una transformación organizacional, ilustrando el valor de las tecnologías de información emergentes; en un sentido prescriptivo, fundamentando casos de negocio dentro de un marco teórico y sugiriendo posibles cursos de acción; y en un sentido dinámico, conceptualizando las interacciones a abordar a través del tiempo (Henderson y Venkatraman, 1992). Por otra parte, si bien diversos estudios reconocen la existencia de un conjunto de antecedentes de la alineación, entre ellos, la cultura corporativa, el conocimiento compartido, y experiencia previa (Chan y Reich, 2007), se debe destacar el trabajo de Gupta y colegas en relación a las capacidades de alineación (Gupta et al., 1997). Los resultados de su trabajo demostraron que la alineación entre la estrategia empresarial y la estrategia de tecnologías de información se puede traducir en un conjunto particular de competencias distintivas en gestión de tecnologías de información para cada tipo de estrategia. En ese sentido, los autores establecieron que no existe una sola solución para problemas de alineación, y más bien, existen capacidades de alineación poseídas por una organización que le permite aprovechar la tecnología para obtener ventajas competitivas. En concreto, no hay una lista de reglas que garanticen el éxito de la alineación, sino que esta alineación depende de la existencia de capacidades instaladas en la empresa.

Diversos estudios han planteado recursos y/o capacidades asociados a los SI/TI. Bharadwaj (2000) señala que las organizaciones poseen recursos de $\mathrm{TI}$ (infraestructura física de $\mathrm{TI}$, recursos humanos de $\mathrm{TI}$ (comprendiendo habilidades de TI técnicas y directivas), y recursos intangibles habilitados por las TI, como por ejemplo, los activos de conocimiento) y una capacidad de TI (entendiendo esta como la habilidad para movilizar y desplegar recursos basados en TI en combinación con otros recursos y capacidades); Dehning y Stratopoulos (2003) consideran que los recursos y capacidades de TI pueden incluir habilidades de TI directivas, habilidades de TI técnicas, e infraestructura de TI; Peppard y Ward (2004) establecen que las organizaciones poseen una capacidad de SI referida a tres elementos inter-relacionados, una fusión de conocimiento de negocio con conocimiento de SI, una plataforma de TI flexible y reusable y un efectivo proceso de uso de SI/TI; Teo y Ranganathan (2003) se refieren a recursos de TI para agrupar a hardware, software, comunicaciones, aplicaciones de TI, y personal de TI, los que pueden mejorar el desempeño de la organización solo si se complementan con otros recursos, específicamente, recursos humanos y recursos de negocio (necesarios para la explotación exitosa de los recursos de TI); autores como Nevo y Wade (2010), Drnevich y Croson, 2013) plantean que los recursos TI son las capacidades de las empresas de utilizar los activos en el ámbito $\mathrm{SI} / \mathrm{TI}$ que disponen, sean tangibles (infraestructura, personal, $\mathrm{SI} / \mathrm{TI}$ ) o intangibles (conocimientos $\mathrm{SI} / \mathrm{TI}$ ) en beneficio del negocio.

Si se considera que los recursos de SI/TI pueden encontrarse, en la actualidad, al alcance de la mayoría de las organizaciones debido a su facilidad de adquisición e imitabilidad, se puede establecer que estos recursos en sí no son suficientes para alcanzar ventajas competitivas sostenibles (Bharadwaj, 2000; Teo y Ranganathan, 2003; Dehning y Stratopoulos, 2003), sino que éstas se alcanzarán dependiendo de la forma en que la organización utiliza tales recursos, lo que está determinado por las "capacidades" que tenga para utilizarlos. Estas "capacidades" constituyen, entonces, un elemento de gran relevancia en el uso de los recursos de SI/TI, si se desea mejorar y mantener la posición competitiva de la organización. Araya et al. (2007b) consideran capacidades asociadas con SI/TI, las que se refieren a todos aquellos elementos o factores, de naturaleza tangible e intangible, que se relacionan o necesitan para que los $\mathrm{SI} / \mathrm{TI}$ sean desarrollados y utilizados en forma eficaz y eficiente, y que se encuentran en el conjunto de la organización, es decir, dentro y fuera del área de $\mathrm{SI} / \mathrm{TI}$. En particular, Araya et al. (2007b) establecen que las capacidades asociadas a los SI/TI contemplan tres tipos de capacidades, las Capacidades Técnicas asociadas con SI/TI, las Capacidades Directivas asociadas con SI/TI, y las Capacidades Organizativas asociadas con SI/TI, como es presentado en la Tabla 1.

Como se ha planteado, un interés prioritario de las empresas es el logro de ventajas competitivas y mejoras en su desempeño, en lo que pueden contribuir los $\mathrm{SI} / \mathrm{TI}$ al estar alineados con la estrategia del negocio definida. En este sentido, la Teoría de Recursos y Capacidades ha planteado que una organización puede poseer capacidades asociadas a los $\mathrm{SI} / \mathrm{TI}$ que permiten contribuir al logro de ventajas competitivas, las que es posible clasificar en Capacidades Técnicas asociadas a SI/TI, Capacidades Directivas asociadas a SI/TI y Capacidades Organizativas asociadas a SI/TI. Luego, si la alineación entre los SI/TI y la estrategia de negocio puede colaborar en el logro de ventajas competitivas, y si en el ámbito de los SI/TI se encuentran Capacidades Asociadas a SI/TI, es importante analizar si dichas capacidades pueden presentar una incidencia positiva sobre el alineamiento mencionado, lo que ha originado la hipótesis de investigación de este estudio, concretamente: H1: Las capacidades asociadas con los SI/TI presentan una incidencia positiva sobre el alineamiento entre los $\mathrm{SI} / \mathrm{TI}$ y la estrategia de negocio. 
Tabla 1: Descripción de capacidades asociadas con SI/TI (Fuente: Araya et al., 2007b)

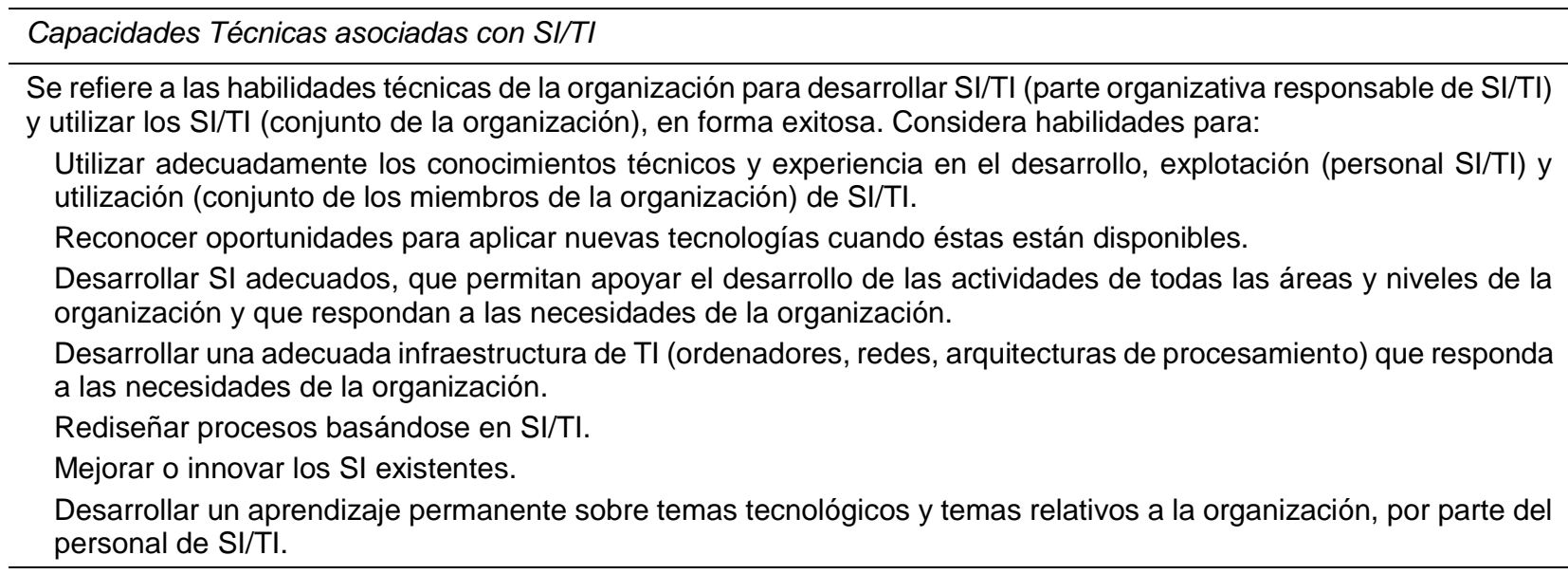
Capacidades Directivas asociadas con SI/TI

Se refiere a las habilidades de la parte directiva de la organización (del área de SI/TI y de las demás áreas y niveles, pero especialmente la primera) para concebir, desarrollar y explotar aplicaciones de SI/TI para apoyar el desarrollo de las actividades y funcionamiento de la organización, en forma exitosa. Considera habilidades para:

Desarrollar una adecuada alineación entre la misión y objetivos de la institución y los SI/TI.

Identificar, priorizar, planificar y liderar proyectos de SI/TI de acuerdo a las necesidades de la institución.

Identificar, planificar, captar, asignar, coordinar y manejar recursos de SI/TI.

Comprometer a los miembros de la organización con el desarrollo y utilización de SI/TI.

Desarrollar un adecuado nivel de conocimientos de la misión y objetivos de la institución, por parte de los directivos de $\mathrm{SI} / \mathrm{TI}$.

Desarrollar un adecuado nivel de conocimientos de SI/TI por parte de los directivos de la organización.

Demostrar un apoyo y compromiso con los SI/TI, por parte de la alta dirección.

Anticiparse a futuras necesidades de SI/TI de la organización (directivos, funcionales y agentes internos y externos), según la misión y objetivos.

Entender y apreciar las necesidades, según la misión y objetivos de la organización, de otros directivos funcionales y agentes internos y externos, por parte de los directivos de SI/TI.

Capacidades Organizativas asociadas con SI/TI

Se refiere a las habilidades de la organización para desarrollar (dentro y fuera del área de $\mathrm{SI} / \mathrm{TI}$ ) aspectos organizativos que pueden facilitar la concepción, desarrollo y explotación de los SI/TI, en forma exitosa. Considera habilidades para:

Concebir, desarrollar y explotar una cultura favorable al entendimiento de la tecnología como apoyo a la misión y objetivos de la institución, por parte del personal de SI/TI.

Concebir, desarrollar y explotar una cultura favorable a los SI/TI (cultura favorable al cambio), por parte de los usuarios.

Concebir, desarrollar y explotar adecuadas relaciones, buena comunicación, cooperación y coordinación entre las personas involucradas con el desarrollo y utilización de SI/TI (especialistas y usuarios).

Concebir, desarrollar y explotar relaciones con agentes externos por medio de los SI/TI.

Concebir, desarrollar y explotar relaciones dentro de la organización por medio de los SI/TI.

\section{METODOLOGÍA}

La validación del modelo propuesto consideró un estudio empírico en la Región del Biobío de Chile, aplicando instrumentos de evaluación a responsables o encargados de SI/TI de empresas, o bien a directivos que, por sus cargos, presentaran alguna influencia en las decisiones relacionadas con los $\mathrm{SI} / \mathrm{TI}$ debido al efecto o impacto que pueden presentar en la organización, obteniéndose datos válidos de 64 empresas presentes en la zona. Las escalas de medida para las variables se basaron en estudios previos. La medición de las variables de capacidades asociadas a SI/TI se basó en Araya (2007), donde se utiliza una escala Likert de cinco puntos para determinar si las capacidades asociadas a SI/TI influyen sobre el desempeño organizativo, mientras que la medición de alineación entre SI/TI y el negocio contempló a Tian et al. (2010), que presenta una escala Likert de cinco puntos para analizar el alineamiento TI con el negocio en el marco de un estudio sobre las capacidades TI de una organización y su posible contribución a la obtención de ventajas competitivas. EI análisis estadístico de los datos se llevó a cabo por medio de modelo de ecuaciones estructurales basado en PLS y utilizando el software SmartPLS (Hair et al., 2014). 


\section{RESULTADOS}

Siguiendo el procedimiento descrito por Hair et al. (2014) para modelos de componentes jerárquicos, se realizó en análisis para el constructo de segundo orden Capacidades Asociadas a SI/TI. Según la aproximación propuesta por esos autores, y dado el carácter reflejo de los indicadores de los constructos de primer orden que forman las Capacidades Asociadas a SI/TI (Capacidades Directivas, Capacidades Organizativas y Capacidades Técnicas), en un primer análisis se determinó la fiabilidad individual de ítems y de constructo para cada uno de ellos, como asimismo su validez convergente y discriminante. El análisis de fiabilidad individual señaló la necesidad de eliminar los ítems $\mathrm{CO} 3$ y $\mathrm{CO} 4$, todos los demás ítems exhiben buena fiabilidad individual. La Tabla 2 muestra las cargas de los ítems del modelo de medida. La fiabilidad de los constructos se evaluó positivamente usando el índice Fiabilidad Compuesta (CR), y por otra parte, la validez convergente se determinó a través de la Varianza Promedio Extraída (AVE), la Tabla 3 muestra estos dos índices. Finalmente, la validez discriminante se valoró a través del índice Ratio Rasgo Hetero - Rasgo Mono (HTMT), para todo los casos bajo el valor 0,9.

Tabla 2: Ítems y cargas de las variables latentes

\begin{tabular}{|c|c|c|c|}
\hline Constructo & Código & Carga & Items \\
\hline \multirow[t]{6}{*}{ Alineamiento } & A1 & 0,88 & Los SI reflejan las metas del plan de negocio \\
\hline & A2 & 0,89 & Los SI reflejan la estrategia del negocio \\
\hline & A3 & 0,75 & Los SI reflejan las fuerzas externas del entorno empresarial \\
\hline & A4 & 0,77 & Los planes del negocio se refieren a planes de los SI \\
\hline & A5 & 0,72 & $\begin{array}{l}\text { Los planes del negocio se refieren a las tecnologías de } \\
\text { información específicas }\end{array}$ \\
\hline & A6 & 0,88 & $\begin{array}{l}\text { Los planes del negocio tienen expectativas razonables de } \\
\text { los SI }\end{array}$ \\
\hline \multirow[t]{5}{*}{$\begin{array}{l}\text { Capacidades } \\
\text { Directivas }\end{array}$} & CD1 & 0,81 & $\begin{array}{l}\text { Los directivos de SI/TI conocen y comprenden la misión y } \\
\text { objetivos de la organización }\end{array}$ \\
\hline & CD2 & 0,71 & $\begin{array}{l}\text { Los directivos de la organización que no son del área de } \\
\text { SI/TI poseen y han desarrollado un adecuado nivel de } \\
\text { conocimientos y experiencia en } \mathrm{SI} / \mathrm{TI}\end{array}$ \\
\hline & CD3 & 0,83 & $\begin{array}{l}\text { La alta dirección de la organización apoya y se compromete } \\
\text { con los proyectos de SI/TI }\end{array}$ \\
\hline & CD4 & 0,71 & $\begin{array}{l}\text { Existe un adecuado nivel de anticipación de futuras } \\
\text { necesidades de SI/TI de la organización (directivos } \\
\text { funcionales y agentes internos y externos), según la misión } \\
\text { y objetivos de la institución }\end{array}$ \\
\hline & CD5 & 0,87 & $\begin{array}{l}\text { Los directivos del área de } \mathrm{SI} / \mathrm{TI} \text { entienden y aprecian las } \\
\text { necesidades de otros directivos funcionales y agentes } \\
\text { internos y externos de la organización }\end{array}$ \\
\hline \multirow[t]{11}{*}{$\begin{array}{l}\text { Capacidades } \\
\text { Organizativas }\end{array}$} & $\mathrm{CO1}$ & 0,71 & $\begin{array}{l}\text { Los miembros de la organización entienden los SI/TI como } \\
\text { un medio para apoyar el cumplimiento de la misión y } \\
\text { objetivos definidos }\end{array}$ \\
\hline & $\mathrm{CO} 2$ & 0,64 & $\begin{array}{l}\text { Los usuarios no presentan actitudes de rechazo o desprecio } \\
\text { por los } \mathrm{SI} / \mathrm{TI} \text { que deben utilizar }\end{array}$ \\
\hline & $\mathrm{CO} 3$ & Eliminado & $\begin{array}{l}\text { Los usuarios y el personal de SI/TI mantienen adecuados } \\
\text { niveles de comunicación, cooperación y coordinación }\end{array}$ \\
\hline & $\mathrm{CO} 4$ & Eliminado & $\begin{array}{l}\text { Se utilizan } \mathrm{SI} / \mathrm{TI} \text { para el desarrollo de una comunicación y } \\
\text { coordinación adecuada con agentes externos (proveedores, } \\
\text { clientes, y otras organizaciones) }\end{array}$ \\
\hline & $\mathrm{CO5}$ & 0,79 & $\begin{array}{l}\text { Se utilizan SI/TI para el desarrollo de una comunicación y } \\
\text { coordinación adecuada dentro de la organización }\end{array}$ \\
\hline & $\mathrm{CO} 6$ & 0,77 & $\begin{array}{l}\text { Se aplican políticas, procedimientos y reglas para la } \\
\text { utilización adecuada de los SI/TI en la organización }\end{array}$ \\
\hline & $\mathrm{CO} 7$ & 0,81 & $\begin{array}{l}\text { Existen procedimientos e instancias para que los usuarios } \\
\text { manifiesten sus inquietudes y necesidades sobre los } \mathrm{SI} / \mathrm{TI}\end{array}$ \\
\hline & $\mathrm{CO8}$ & 0,83 & $\begin{array}{l}\text { Se ha definido y desarrollado una adecuada alineación } \\
\text { entre la misión y objetivos de la organización y los SI/TI }\end{array}$ \\
\hline & CO9 & 0,85 & $\begin{array}{l}\text { Se ha llevado a cabo proceso minucioso y ordenado de } \\
\text { identificación, priorización, planificación y ejecución de } \\
\text { proyectos de SI/TI de acuerdo a las necesidades de la } \\
\text { organización }\end{array}$ \\
\hline & CO10 & 0,85 & $\begin{array}{l}\text { Se ha llevado a cabo un proceso minucioso y ordenado de } \\
\text { identificación, planificación, captación, asignación y } \\
\text { coordinación de recursos de SI/TI }\end{array}$ \\
\hline & CO11 & 0,84 & $\begin{array}{l}\text { La organización se encuentra comprometida con el } \\
\text { desarrollo y utilización de SI/TI }\end{array}$ \\
\hline
\end{tabular}


Tabla 2 (continuación)

\begin{tabular}{|c|c|c|c|}
\hline Constructo & Código & Carga & Ítems \\
\hline \multirow[t]{9}{*}{$\begin{array}{l}\text { Capacidades } \\
\text { Técnicas }\end{array}$} & CT1 & 0,83 & $\begin{array}{l}\text { El área de SI/TI posee y ha desarrollado los conocimientos } \\
\text { y experiencia técnica adecuados para el desarrollo y } \\
\text { explotación de SI (por ejemplo, lenguajes de programación, } \\
\text { sistemas operativos, etc.) }\end{array}$ \\
\hline & CT2 & 0,71 & $\begin{array}{l}\text { En la organización existen y se han desarrollado los } \\
\text { conocimientos y experiencia técnica adecuados para utilizar } \\
\text { los SI correctamente, por parte del conjunto de sus } \\
\text { miembros }\end{array}$ \\
\hline & CT3 & 0,72 & $\begin{array}{l}\text { Se reconocen oportunidades para aplicar nuevas } \\
\text { tecnologías cuando se encuentran disponibles }\end{array}$ \\
\hline & CT4 & 0,81 & $\begin{array}{l}\text { Se han desarrollado SI adecuados, que permiten apoyar el } \\
\text { desarrollo de las actividades de todas las áreas y niveles de } \\
\text { la organización y que responden a las necesidades de la } \\
\text { organización }\end{array}$ \\
\hline & CT5 & 0,69 & $\begin{array}{l}\text { Se ha desarrollado una adecuada infraestructura de } \mathrm{TI} \\
\text { (ordenadores, redes, arquitecturas de procesamiento) que } \\
\text { responden a las necesidades de la organización }\end{array}$ \\
\hline & CT6 & 0,77 & Se han rediseñado procesos basándose en $\mathrm{SI} / \mathrm{TI}$ \\
\hline & CT7 & 0,89 & Se desarrollan mejoras o innovaciones en los SI existentes \\
\hline & CT8 & 0,80 & $\begin{array}{l}\text { El área de } \mathrm{SI} / \mathrm{TI} \text { desarrolla un aprendizaje permanente } \\
\text { sobre temas tecnológicos para mejorar y actualizar sus } \\
\text { conocimientos }\end{array}$ \\
\hline & CT9 & 0,77 & $\begin{array}{l}\text { El área de } \mathrm{SI} / \mathrm{TI} \text { desarrolla un aprendizaje permanente } \\
\text { sobre temas relativos a la organización para mejorar y } \\
\text { actualizar sus conocimientos y entendimiento de ésta }\end{array}$ \\
\hline \multirow{4}{*}{$\begin{array}{l}\text { Capacidades } \\
\text { Asociadas a } \\
\text { SI/TI }\end{array}$} & C1 & 0,95 & Puntuación calculada a partir de los ítem de Capacidades \\
\hline & & & Técnicas \\
\hline & $\mathrm{C} 2$ & 0,85 & $\begin{array}{l}\text { Puntuación calculada a partir de los ítem de Capacidades } \\
\text { Organizativas }\end{array}$ \\
\hline & $\mathrm{C} 3$ & 0,89 & $\begin{array}{l}\text { Puntuación calculada a partir de los ítem de Capacidades } \\
\text { Directivas }\end{array}$ \\
\hline
\end{tabular}

Probada la bondad del modelo de medida asociado a los constructos de primer orden, se procedió a calcular las puntuaciones estandarizadas que los representan en el constructo de segundo orden (ver Tabla 2). A continuación se evaluó con éxito la escala de medida del constructo Alineamiento, sus cargas pueden ser vistas en la Tabla 2 y los coeficientes asociados a su fiabilidad de constructo y validez convergente en la Tabla 3. Dado que el constructo Capacidades Asociadas a SI/TI es de tipo formativo no se debió probar la validez discriminante entre este y el constructo Alineamiento.

Tabla 3: Coeficientes del modelo de medida

\begin{tabular}{lcc}
\hline Constructo & $\begin{array}{c}\text { Fiabilidad } \\
\text { Compuesta (CR) }\end{array}$ & $\begin{array}{c}\text { Varianza Promedio } \\
\text { Extraída (AVE) }\end{array}$ \\
\hline Capacidades Directivas & 0,89 & 0,62 \\
Capacidades Organizativas & 0,94 & 0,62 \\
Capacidades Técnicas & 0,93 & 0,61 \\
Alineamiento & 0,92 & 0,67 \\
Capacidades Asociadas a SI/TI & 0,96 & 0,53 \\
\hline
\end{tabular}

Finalmente, se procedió a analizar el modelo estructural que asocia las Capacidades Asociadas a SI/TI con Alineamiento. La Tabla 4 muestra los índices de ajuste de este modelo, dado estos valores se acepta que el modelo se ajusta a los datos, es decir, los datos no contienen más información que la que el modelo transmite. La Tabla 5 muestra el resultado del modelo estructural, este análisis señala que la variable dependiente medida por el constructo Alineamiento es explicada un $52 \%$ por la variable independiente medida por el constructo de segundo orden Capacidades Asociadas a SI/TI. El coeficiente estandarizado beta con un valor de 0,72 es significativo (valor $p<0,001,{ }^{* * *}$ ), el análisis de la significación de este coeficiente se realizó con la técnica no paramétrica bootstrapping con 5000 sub muestras. Este resultado permite soportar la hipótesis $\mathrm{H} 1$.

Tabla 4: Índices de ajuste del modelo

\begin{tabular}{lr}
\hline Índice & \multicolumn{1}{c}{ Valor } \\
\hline Raíz cuadrada media residual normalizada (SRMR) & 0,085 \\
Índice de ajuste normado (NFI) & 0,862 \\
Chi-cuadrado & 59,067 \\
\hline
\end{tabular}


Tabla 5: Resultados del modelo estructural

\begin{tabular}{ccccc}
\hline $\begin{array}{c}\text { Variable } \\
\text { Dependiente }\end{array}$ & $\begin{array}{c}\text { Variable } \\
\text { Independiente }\end{array}$ & $R^{2}$ & Beta & Sig. \\
\hline Alineamiento & Capacidades Asociadas a SI/TI & & 0,52 & \\
& Cand & \\
\hline
\end{tabular}

\section{DISCUSIÓN}

Deseamos destacar tres elementos del resultado de este estudio. Primero, las escalas de medida para las variables del modelo son altamente fiables (una de 0,89 y el resto sobre 0,9 ), esto posibilita la realización de indagaciones futuras sobre el fenómeno en un contexto latinoamericano. Segundo, en relación a capacidades asociadas a SI/TI, los resultados muestran que las tres dimensiones que la forman tienen un peso importante en su determinación. En este sentido, entre las capacidades directivas se destaca que los directivos SI/TI deben apoyar y comprometerse con las necesidades de otros directivos de la empresa, así como comprender la misión y los objetivos de la organización. Por otra parte, entre las capacidades organizativas se subraya el compromiso necesario de la organización con el desarrollo y utilización de los SI, y la necesidad de llevar a cabo un proceso minucioso y ordenado de identificación, planificación, ejecución y asignación de recursos de proyectos de $\mathrm{SI} / \mathrm{TI}$, en consonancia con las necesidades de la empresa. Y finalmente, asociado las capacidades técnicas, se enfatiza la preparación y actualización permanente de los profesionales del área de $\mathrm{SI} / \mathrm{TI}$.

Y Tercero, si bien el alineamiento es explicado por capacidades asociadas a SI/TI, creemos que el valor del coeficiente de determinación podría estar parcialmente determinado por variables no consideradas en el estudio, como es el caso de los tipos de estrategias de negocio (Croteau y Bergeron, 2001; Antosz y Pozo, 2015) o el nivel de desarrollo tecnológico de la empresa (Croteau y Bergeron, 2001; Almazan, Tovar y Quintero, 2017), ya que las capacidades asociadas a SI/TI que la organización posee o ha construido pueden depender de la forma de implementación del desarrollo de SI/TI o del nivel de despliegue tecnológico de SI/TI que ha alcanzado la organización, lo que podría estar sujeto al tipo de estrategia de negocio que se ha puesto en marcha para el logro de un mejor desempeño o de ventajas competitivas. En este sentido, el nivel de desarrollo de SI/TI en la organización puede afectar la capacidad de maximizar la flexibilidad de las plataformas de SI/TI disponibles, facilitando o limitando la posibilidad de dar respuesta rápida a amenazas y oportunidades de mercado, lo que podría favorecer o disminuir la mantención de la alineación entre la estrategia de negocio y los SI/TI (Reynolds y Yetton, 2015). Si bien este estudio ha permitido obtener una visión sobre el efecto o relación entre capacidades asociadas con SI/TI y el alineamiento de estos últimos con el negocio, se ha considerado solo a empresas presentes en la Región de Biobío (Chile), lo que reduce la cobertura geográfica a la realidad de una zona específica de un país determinado, constituyendo una limitación. Sin embargo, esta restricción representa una oportunidad de efectuar nuevos estudios, concernientes a otras zonas geográficas, lo que permitiría enriquecer aún más el conocimiento sobre el impacto de la utilización de los SI sobre los resultados obtenidos por las organizaciones.

\section{CONCLUSIONES}

Este estudio muestra que las capacidades asociadas con SI/TI inciden en el alineamiento de $\mathrm{SI} / \mathrm{TI}$ y el negocio, donde pueden intervenir aspectos directivos, organizativos y técnicos relacionados con SI/TI. La originalidad de estos hallazgos está asociada a soportar la validez de esta propuesta en un contexto de desarrollo tecnológico no avanzado.

Si bien el desarrollo e implementación de sistemas y tecnologías de información requiere el apoyo de la alta dirección, se aprecia como relevante la participación de los directivos $\mathrm{SI} / \mathrm{TI}$, relacionada con su apoyo y compromiso con las necesidades informáticas de otros directivos de la empresa y su nivel de asimilación de la misión y objetivos de la organización. Lo anterior apunta al entendimiento que los SI/TI no constituyen un fin en sí mismos, sino un medio para apoyar el logro de los propósitos institucionales.

\section{REFERENCIAS}

Almazán, D.A., Y.S. Tovar y J.M.M. Quintero, Influencia de los sistemas de información en los resultados organizacionales, Contaduría y administración, 62(2), 303-320 (2017)

Antosz, M. S. y J. M. Pozo, Análisis de estrategias y modelos de aplicación de las TIC en las empresas, Revista Científica Ecociencia, 2 (6) (2015)

Araya, S., J. Chaparro, A. Orero, H. Joglar, Sistemas y Tecnologías de Información y su relación con el Desempeño de Instituciones Universitarias en el ámbito de Gestión Institucional: un estudio desde la Teoría de Recursos y Capacidades. In International Conference on Industrial Engineering \& Industrial Management, 1805-1816 (2007a) 
Araya, S., J. Chaparro, A. Orero y H. Joglar, An integrative view of IS/IT and organizational resources and capabilities, Issues in Informing Science \& Information Technology, 4, 629-640 (2007b)

Barney, J., M. Wright y D.J. Ketchen, The resource-based view of the firm: Ten years after 1991, Journal of management, 27(6), 625-641 (2001)

Bharadwaj, A. S., A resource-based perspective on information technology capability and firm performance: an empirical investigation, MIS Quarterly, 24 (1), 169-196 (2000)

Chan, Y. E. y B. H. Reich, IT alignment: what have we learned? Journal of Information Technology, 22(4), 297-315 (2007)

Chan, Y., S. Huff, D. Barclay y D. Copeland, Business strategic orientation, information systems strategic orientation, and strategic alignment, Information Systems Research, 8 (2), 125-150 (1997)

Cragg, P., M. King y H. Hussin, IT alignment and firm performance in small manufacturing firms, Journal of Strategic Information Systems, 11, 109-132 (2002)

Croteau, A. y F. Bergeron, An Information Technology Trilogy, Business Strategy, Technological Deployment and Organizational Performance, Journal of Strategic Information Systems, 10(2), 77-99 (2001)

Dehning, B. y T. Stratopoulos, Determinants of a sustainable competitive advantage due to an IT-enabled strategy, The Journal of Strategic Information Systems, 12 (1), 7-28 (2003)

Drnevich, P.L. y D.C. Croson, Information Technology and Business-level strategy: Toward an integrated Theoretical Perspective, MIS Quarterly, 37(2), 483-509 (2013)

Gu, J.W. y H. W. Jung, The effects of IS resources, capabilities, and qualities on organizational performance: An integrated approach, Information \& Management, 50(2-3), 87-97 (2013)

Gupta, Y. P., J. Jarimi y T. M. Somers, Alignment of a firm's competitive strategy and information technology management sophistication: the missing link, IEEE Transactions on Engineering Management, 44(4), 399-413 (1997)

Hair, J.F., G.T. Hult, C.M. Ringle y M. Sarstedt, A primer on partial least squares structural equation modeling (PLS-SEM), 2nd Ed., Thousand Oaks, Sage (2014)

Henderson, J.C. y N. Venkatraman, Strategic alignment: a model for organizational transformation through information technology, Transforming Organizations, 97-117 (1992)

Kearns, G. y A. Lederer, The effect of strategic aligment on the use of IS-based resources for competitive advantage, The Journal of Strategic Information Systems, 9 (4), 265-293 (2000)

Lin, H.K., T. L. Chuang, e I. Lin, Elucidating the role of IT/IS assessment and resource allocation in IT/IS performance in hospitals, Information \& Management, 51(1), 104-112 (2014)

Liu, H., W. Ke y K. K. Wei, The impact of IT capabilities on firm performance: The mediating roles of absorptive capacity and supply chain agility, Decision Support Systems, 54(3), 1452-1262 (2013)

Nevo, S. y M. Wade, The Formation and Value of IT-Enabled Resources: Antecedents and Consequences of Synergistic Relationships, MIS Quarterly, 34(1), 163-183 (2010)

Peppard, J. y J. Ward, Beyond strategic information systems: towards an IS capability, Journal of Strategic Information Systems, 13, 167-194 (2004)

Porter, M., Competitive Strategy: Techniques for Analyzing Industries and Competitors, New York, Free Press (1980)

Ramírez-Correa, P. y J. Alfaro-Pérez, El nivel de la inversión en tecnología de información no afecta el rendimiento empresarial: evidencia empírica de las industrias manufactureras chilenas, Journal of Technology Management \& Innovation, 6(4), 225-242 (2011)

Reich, B. H. e I. Benbasat, Measuring the linkage between business and information technology objectives, MIS Quarterly, 20 (1), 55-81 (1996)

Reynolds, P. y P. Yetton, Aligning business and IT strategies in multi-business organizations, Journal of Information Technology, 30(2), 101-118 (2015)

Teo, T. y C. Ranganathan, Leveraging IT resources and capabilities at the housing and development board, The Journal of Strategic Information Systems, 12 (3), 229-249 (2003)

Tian, J., K. Wang, Y. Cheng y B. Johansson, From IT deployment capabilities to competitive advantage: An exploratory study in China, Information Systems Frontiers, 12(3), 239-255 (2010)

Turban, E., L. Volonino y G. Wood, Information Technology For Management: Digital Strategies for Insight, Action, and Sustainable Performance, Wiley Publishing (2015)

Uwizeyemungu, S. y L. Raymond, Impact of an ERP system's capabilities upon the realization of business value: a resource-based perspective, Information Technology \& Management, 13(2), 69-90 (2012)

Valencia, F., C. Marulanda y M. López, Gobierno de las Tecnologías de la Información. Uso y Prácticas en las Entidades Públicas del Triángulo del Café, Colombia, Información Tecnológica, 29(3), 249-256 (2018) 